\title{
The HAP2 subunit of yeast CCAAT transcriptional activator contains adjacent domains for subunit association and DNA recognition: model for the HAP2/3/4 complex
}

\author{
James T. Olesen and Leonard Guarente \\ Department of Biology, Massachusetts Institute of Technology, Cambridge, Massachusetts 02319 USA
}

\begin{abstract}
Budding yeast HAP2 is required in concert with HAP3 and HAP4 to form a heterotrimeric CCAAT-binding transcriptional activation complex at the UAS2 element of CYC1. Functional homologs of HAP2 and HAP3 have been conserved in HeLa cells where HAP2 activity corresponds to a chromatographic fraction designated CP1B. Here, we describe deletion and codon insertion mutagenesis of the Saccharomyces cerevisiae HAP2 subunit. DNA binding was assessed both in vitro and in vivo whereas subunit association and transcriptional activation were analyzed in vivo by using a bifunctional lexA-HAP2 fusion. The results indicate that the 265amino-acid HAP2 protein contains an "essential core" of 65 amino acids, which is entirely sufficient for assembly and DNA binding of the HAP2/3/4 complex. The essential core can be divided further into a region of 44 amino acids, which is sufficient for subunit association, and a region of 21 amino acids, which is required specifically for DNA recognition. The remainder of the HAP2 protein is dispensable. The roles of HAP3 and HAP4 in the heterotrimeric complex are also assessed by using lexA fusions and a fusion of the GAL4 acidic activation domain to HAP2, which partially bypasses a deletion of HAP4 but not HAP3. These data indicate that subunits HAP2 and HAP3 are primarily responsible for site-specific DNA binding by the complex, whereas the HAP4 subunit provides the primary transcriptional activation domain. A model for the function of this regulated transcriptional activation complex is presented.
\end{abstract}

[Key Words: Yeast transcription; CCAAT element; HAP2/3/4; heteromeric complex; Saccharomyces cerevisiae] Received May 4, 1990; revised version accepted July 18, 1990.

Transcriptional activators affect gene expression by first binding to specific DNA sequences near genes and then promoting the initiation of transcription by RNA polymerase II via the various basic transcription factors. Analysis of several eukaryotic transcriptional activators has revealed that they commonly possess a modular structure consisting of separate and independent DNAbinding and transcriptional activating regions (Brent and Ptashne 1985). Site-specific DNA binding has been shown to occur through various common DNA-binding motifs such as the helix-turn-helix (for review, see Pabo and Sauer 1984; Brennan and Matthews 1989|, zinc finger (for review, see Klug and Rhodes 1987; Berg 1989) leucine zipper (Landschulz et al. 1988), and helix-loophelix (Murre et al. 1989a,b) structures. Less is known about the specific structure of transcriptional activating regions, except that such sequences are often enriched in acidic amino acids (Ma and Ptashne 1987), glutamines (Courey and Tjian 1988), or prolines (Mermod et al. 1989).

Most transcriptional activators possess both DNA- binding and transcriptional activating functions in a single polypeptide. Recently, however, complex transcriptional activators consisting of two or three different protein subunits have been recognized and characterized. For example, the genetically defined HAP2 and HAP3 (Olesen et al. 1987) and, later, HAP4 (Forsburg and Guarente 1989a) proteins have been shown to bind as a complex heterotrimeric structure to the sequence CCAAT located in the UAS2UP1 element of Saccharomyces cerevisiae CYC1. Mutations in any one of these subunits abolish transcriptional activation from UAS2. The HAP2/3/4 heterotrimer also activates transcription through CCAAT "boxes" located upstream of HEM1 (Keng and Guarente 1987), as well as COX4 and CYT1 (Schneider 1989). All of these genes encode components of the mitochondrial electron transport chain. As a consequence, hap2, hap3, and hap4 mutant yeast strains fail to express such genes adequately, resulting in an inability to grow on nonfermentable carbon sources where oxidative phosphorylation is essential for energy production. The primary induction of UAS2 (CCAAC) of 
CYC1, or other genes regulated by the complex, occurs when cells are shifted from a fermentable carbon source such as glucose to medium containing a nonfermentable carbon source, such as lactate. The precise mechanism by which this regulation occurs is not fully understood, although changes in the amount of HAP2/3/4 CCAATbinding activity clearly play a major role (Olesen and Guarente 1987). An additional form of UAS2 (CCAAC) regulation is the modulation of HAP2/3/4 activity by heme. In heme-deficient cells, HAP2/3/4 activity from UAS2 is tenfold lower than in cell synthesizing heme. This form of regulation is thought to make the CCAATresponsive respiratory genes sensitive to the availability of environmental oxygen, which is required in the biosynthesis of heme (for review, see Forsburg and Guarente 1989b).

Remarkably, the HAP2/3/4 CCAAT-binding complex appears to have been conserved over the millions of years of evolutionary divergence between yeast and man. Chromatographic fractionation of human cells has identified a similar multicomponent CCAAT-binding complex (Chodosh et al. 1988a). Cooperative binding between the human and yeast subunits has demonstrated that the HAP2 human homolog is contained in a fraction designated $\mathrm{CP} 1 \mathrm{~B}$, whereas the human homolog of HAP3 occurs in fraction CP1A (Chodosh et al. 1988b). These studies revealed that both protein-protein interactions between the subunits and protein-DNA interactions allowing site-specific binding have been conserved between yeast and man by the subunits of this complex transcriptional activator. More recently, the heterodimeric structure of mammalian Fos-Jun has been analyzed in detail (for review, see Curran and Franza 1988), and MyoD1/E12 and other helix-loop-helix protein heterodimers have also been described (Murre et al. 1989b).

None of the subunits of the HAP2/3/4 complex possesses any homology to known DNA-binding motifs; therefore, the subunits and protein domains involved are not apparent. Subsequent to binding to the site specifically, the HAP2/3/4 complex must promote transcription of the downstream gene-presumably by contacting the transcriptional machinery. Interestingly, two of the subunits possess features found in known transcriptional activating regions. HAP2 contains a polyglutamine stretch similar to the transcriptional activating sequences of mammalian SP1 (Courey and Tjian 1988) and also found in numerous Drosophila developmental genes (Wharton et al. 1985), whereas HAP4 contains an essential highly acidic carboxy-terminal region that is interchangeable in function with the acidic transcriptional activation domain of GAL4 (Forsburg and Guarente 1989a). In addition to DNA binding and transcriptional activation functions, each subunit of the HAP2/3/4 heterotrimer must contain surfaces that allow association with the other subunits of the complex, "heterodimerization domains." Indeed, the available evidence suggests that the HAP2, HAP3, and HAP4 subunits are associated together in solution and do not need to be bound to DNA to form the requisite protein- protein interactions for assembly of the complex (Hahn and Guarente 1988, and this paper). Here, we describe experiments that begin a molecular dissection of the HAP2/3/4 complex and the HAP2 subunit, in particular.

\section{Results}

The HAP2 protein contains an essential core of 65 amino acids

Mapping sequences in HAP2 required for DNA binding by $H A P 2 / 3 / 4$ The HAP2 protein is 265 amino acids long and possesses two interesting features in its sequence. One is a repeat of 14 glutamines in a row, extending from amino acid 120 to amino acid 133. The other is a highly basic $(27 \%$ lysine or arginine) amino acid content in the carboxy-terminal 100 amino acids. A similarly charged region of GCN4 has been shown to possess sequence-specific DNA-binding activity (Hope and Struhl 1986), and comparable basic protein domains appear to be involved in DNA recognition by the FosJun family of proteins, as well as MyoD1 and related proteins (see Kouzarides and Ziff 1989). To determine the minimal region of HAP2 required for sequence-specific DNA binding, a series of amino-terminal and carboxy-terminal deletions of the HAP2 protein were synthesized by in vitro transcription of deleted HAP2 templates with SP6 RNA polymerase. The resulting RNA was translated in vitro in a rabbit reticulocyte lysate as described in Materials and methods. Because HAP2 binds to the UAS2UP1 CCAAT box as a complex with HAP3 (Olesen et al. 1987) and HAP4 (Forsburg and Guarente 1989a), regions of the HAP2 protein required for DNA binding may be involved in either subunit association or direct contact with the DNA double helix or both protein-protein and protein-DNA interactions.

Full-length HAP2 protein was generated from the template pSP64: HAP2, which contains the SP6 promoter cloned upstream of the 265 amino acid HAP2 open reading frame (ORF). Gel shift DNA-binding assays performed with in vitro-translated unlabeled HAP2 protein and radiolabeled UAS2 probes revealed the presence of a UAS2UP1 DNA-binding activity (Fig. 1, lane 4), which was absent with a UAS2 probe containing a nonconsensus CCAAC sequence (lane 3). Binding of HAP2 in the absence of exogenously supplied HAP3 and HAP4 is presumably due to the presence of the evolutionarily conserved CP1A activity in the mammalian reticulocyte lysate. The endogenous CA1A/CP1B activity was evident as a faint band in the mock translation control (lane 2). THE CP1A-like activity must be limiting for binding when HAP2 is synthesized in vitro, because adding purified CP1A from HeLa cells to HAP2 reticulocyte translation resulted in a large increase in CCAAT DNAbinding activity (cf. lanes 4 and 6). In the presence of an adequate amount of competitor nucleic acid and supplemental HeLa CP1A, binding of the in vitro-synthesized HAP2 protein was readily evident without interference from the CP1 CCAAT-binding activity endogenous to the rabbit reticulocyte lysate (cf. lanes 5 and 6). Further- 

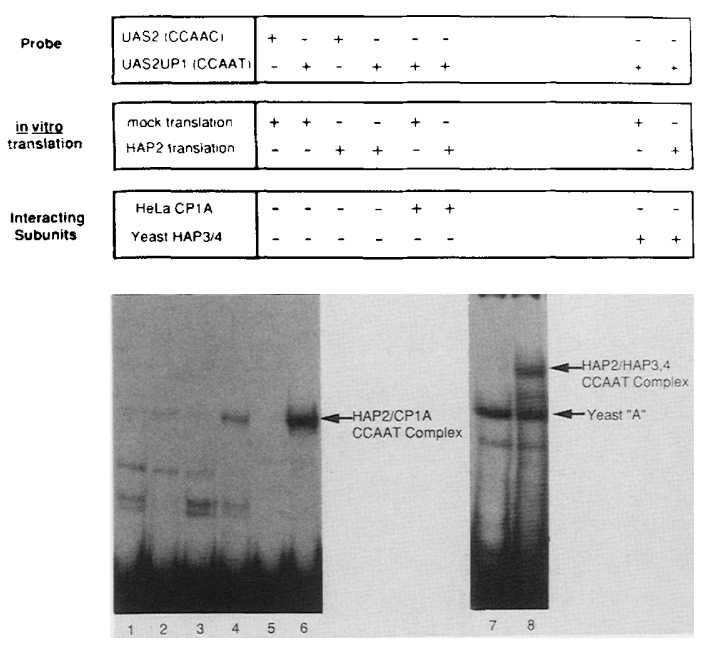

Figure 1. Detection of DNA-binding activity from in vitrosynthesized HAP2. Radiolabeled UAS2 and UAS2UP1 probes were utilized as indicated in gel mobility-shift DNA-binding assays with nonradiolabeled mock or HAP2 in vitro translation products prepared as described in Materials and methods. Lanes $1-4$ contain $0.5 \mu \mathrm{g}$ single-stranded (salmon sperm) DNA and $0.5 \mu \mathrm{g}$ of poly[d(I-C)/d(I-C)] competitor nucleic acid. The HAP2 CCAAT-binding activity detected in lane 4 reflects the presence of a CP1A-like activity endogenous to the rabbit reticulocyte lysate. Detection of the HAP2/3/4 CCAAT DNA-binding activity was facilitated by the addition of more competitor nucleic acid $[3 \mu \mathrm{g}$ of single-stranded DNA and $3 \mu \mathrm{g}$ of poly[d(I-C)/ $\mathrm{d}(\mathrm{I}-\mathrm{C}) \mathrm{and}$ either HeLa CP1A fraction (lanes 5 and 6 ), or a yeast HAP3/HAP4-containing extract (lanes 7 and 8).

more, the in vitro-synthesized HAP2 was also capable of interacting with HAP3 and HAP4 supplied from a crude yeast extract to form the HAP2/3/4/ CCAAT-binding species (cf. lanes 7 and 8). We conclude that this in vitro assay accurately reflects the DNA-binding properties of yeast HAP2.

A series of amino-terminally deleted HAP2 proteins was generated by construction of in-frame deletions of HAP2 by use of either the wild-type (amino acid 1) or a downstream (amino acid 43) ATG initiation codon. Synthesis of the expected size protein was confirmed by translation of a small amount of the RNA in the presence of $\left[{ }^{35} S\right]$ methionine and molecular weight analysis of the translation products on an SDS-PAGE gel, as described in Materials and methods (results not shown). The remaining RNA was translated in the presence of unlabeled methionine, and the resulting unlabeled HAP2 protein was examined for site-specific DNA binding in the gel retardation assay. Binding of the amino-terminal deletion proteins to UAS2UP1 is shown in Figure 2A, and results are summarized in Figure 2B. Deletions extending to amino acid 154 (lanes 1-7) did not disrupt DNA binding, although they increased the mobility of the complex, as would be expected from the decrease in molecular weight of the HAP2 component. Deleting further to amino acid 169 resulted in loss of CCAAT-binding activity (lane 8). Thus, the first 153 amino acids of HAP2, including the polyglutamine stretch, are not required for site-specific DNA binding.
Carboxy-terminal deletions of HAP2 were generated by truncation of pSP64 : HAP2 template at a series of restriction sites in the ORF. As shown in Figure $2 \mathrm{~A}$ and summarized in Figure 2B, deletions up to amino acid 218 were still able to bind site specifically (lanes 9-12). Truncating further to amino acid 197 obliterated binding (lane 13). Furthermore, to confirm that there was no redundancy in DNA-binding function of the amino- and carboxy-dispensable sequences, truncations of $\Delta \mathrm{N} 154$ were generated (lanes 18-24). The 65-amino-acid region extending from 154 to 218 was capable of forming a CCAAT-binding complex ( $\triangle$ N154 2 C218, lane 22), demonstrating that the entire DNA-binding domain of HAP2 is contained within this region. Furthermore, representative HAP2 deletions were also examined for cooperative CCAAT-binding activity with yeast HAP2/HAP4 (assays not shown). The results, summarized in Figure 2B, indicate that the region extending from amino acid 154 to amino acid 218 is adequate for the formation of a CCAAT-binding complex with either mammalian CP1A or yeast HAP3/HAP4. We conclude that these residues constitute a core of the HAP2 protein, which makes the necessary protein-DNA and/or protein-protein interactions sufficient to assembly HAP2 into a heterotrimeric CCAAT-binding complex.

Codon insertion mutations in the DNA-binding core domain obliterate HAP2 function in vivo As a first step toward determining the regions of HAP2 required for its in vivo function, we introduced a total of 20 codon insertion mutations throughout the HAP2 protein (Barany 1985). Net insertions from 1 to 4 amino acids and a single net deletion of 3 amino acids were constructed primarily by introduction of linkers at specific restriction sites such that the reading frame of the HAP2 protein was maintained (see Materials and methods).

The amino acid sequence changes made are summarized in Figure 3A. The mutations are named according to the amino acid residue(s) and codon position(s) eliminated by the change, followed by a slash and the amino acids inserted at that site. The ability of the resulting HAP2 mutant proteins to support growth on lactate and to activate a UAS2-lacZ fusion is summarized in Figure 3B. Only two of the mutations-13 (Y168/SRD) and 15 (G209/AGSR)-affected complementation of a hap2 null mutation. Both of these codon insertions occur in the HAP2 DNA-binding core domain defined above.

Interestingly, mutant 14 (K180-184/DP), which occurs between these two mutations and is in the center of the DNA-binding domain, had no apparent phenotype. $\mathrm{Mu}$ tants $4,6,7,8$, and 11-20 fully complemented the hap2 mutant. We conclude that in vivo function of the HAP2 protein is quite insensitive to codon insertion mutations occurring outside of the core region required for site-specific DNA binding.

Deletion mutations reveal that the core domain is sufficient for HAP2 function in vivo To determine whether the regions of HAP2 not required for site-specific DNA binding and not affected by codon insertion mutagenesis are essential to the functioning of the protein in vivo, we 

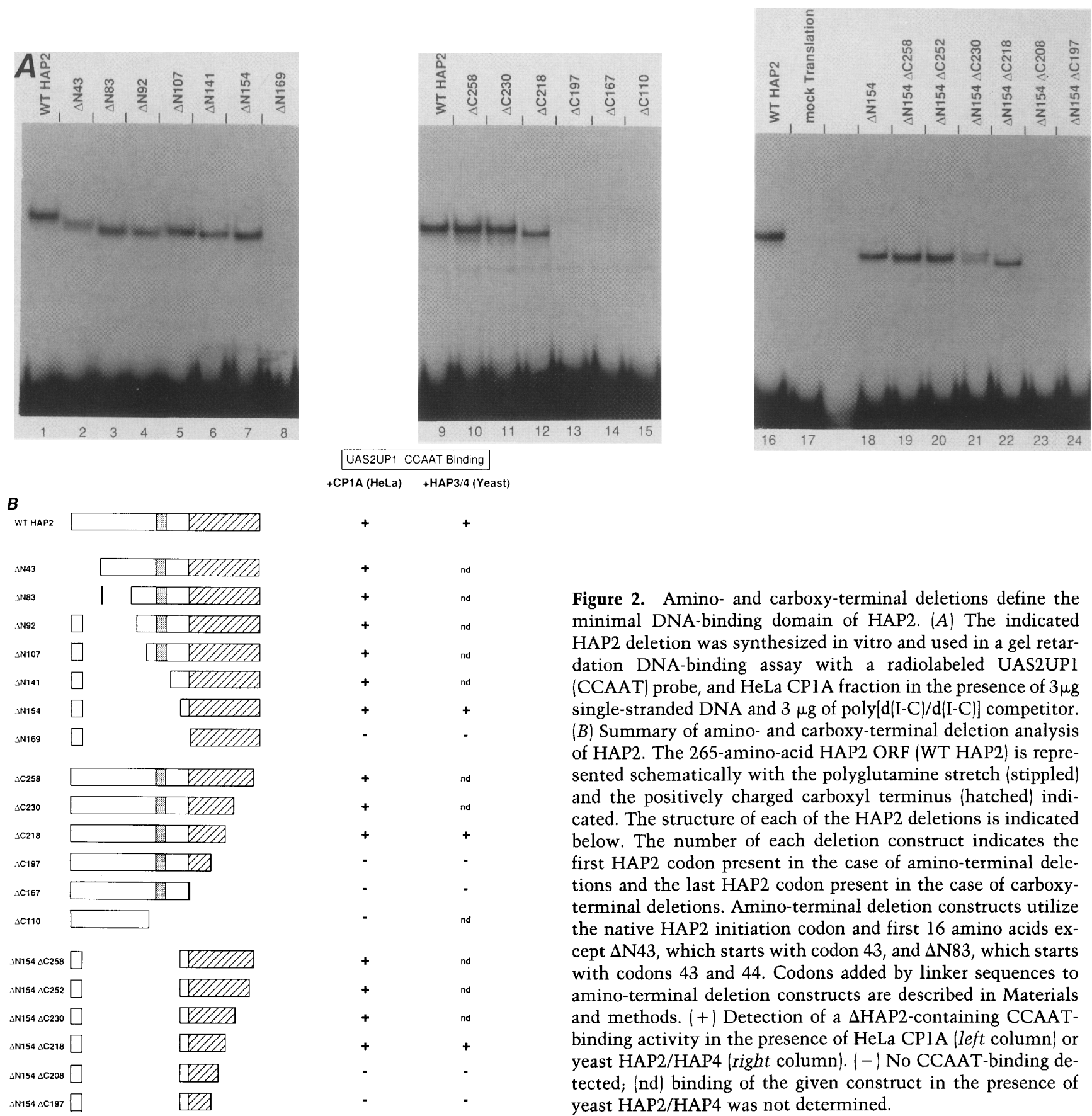

Figure 2. Amino- and carboxy-terminal deletions define the minimal DNA-binding domain of HAP2. (A) The indicated HAP2 deletion was synthesized in vitro and used in a gel retardation DNA-binding assay with a radiolabeled UAS2UP1 (CCAAT) probe, and HeLa CP1A fraction in the presence of $3 \mu \mathrm{g}$ single-stranded DNA and $3 \mu \mathrm{g}$ of poly $[\mathrm{d}(\mathrm{I}-\mathrm{C}) / \mathrm{d}(\mathrm{I}-\mathrm{C})]$ competitor. (B) Summary of amino- and carboxy-terminal deletion analysis of HAP2. The 265-amino-acid HAP2 ORF (WT HAP2) is represented schematically with the polyglutamine stretch (stippled) and the positively charged carboxyl terminus (hatched) indicated. The structure of each of the HAP2 deletions is indicated below. The number of each deletion construct indicates the first HAP2 codon present in the case of amino-terminal deletions and the last HAP2 codon present in the case of carboxyterminal deletions. Amino-terminal deletion constructs utilize the native HAP2 initiation codon and first 16 amino acids except $\Delta \mathrm{N} 43$, which starts with codon 43 , and $\Delta N 83$, which starts with codons 43 and 44 . Codons added by linker sequences to amino-terminal deletion constructs are described in Materials and methods. $(+)$ Detection of a $\triangle$ HAP2-containing CCAATbinding activity in the presence of HeLa CPlA (left column) or yeast HAP2/HAP4 (right column). (-) No CCAAT-binding detected; (nd) binding of the given construct in the presence of yeast HAP2/HAP4 was not determined.

constructed carboxy- and amino-terminal deletions of the HAP2 proteins as fusions to the bacterial repressor lexA (see Materials and methods). As reported earlier (Pinkham et al. 1987), the lexA-HAP2 fusion protein is capable of activating transcription from a yeast promoter containing the lexA operator but no yeast upstream promoter elements. This fusion protein also fully complements a hap2 null mutation for growth on lactate. The lexA-HAP2 fusion is thus bifunctional in that it binds site specifically at both the lexA operator and at the UAS2UP1 CCAAT box as a complex with the HAP3 and HAP4 subunits. Figure 4A summarizes the phenotypes

observed for amino-terminal deletions of HAP2 fused to lexA. In this section, we discuss complementation of the hap2 null mutant for growth on lactate by these mutant lexA-HAP2 constructs. Deletions extending to amino acid 154 (mutants $\mathrm{lx} \Delta \mathrm{N} 45-\mathrm{l} \mathrm{x} \Delta \mathrm{N} 154$ ) did not affect complementation. As expected, further deletions that impinge on the DNA-binding domain of HAP2 (mutants $\mathrm{l} x \Delta \mathrm{N} 169-\mathrm{l} \Delta \Delta \mathrm{N} 255)$ abolished complementation.

Figure 4B shows the results of carboxy-terminal deletion mutagenesis of the lexA-HAP2 fusion. Deletions extending to the carboxyl end of the DNA-binding domain (amino acid 218, mutant $\mathrm{lx} \Delta \mathrm{C} 218$ ) did not detect- 
A

\begin{tabular}{|c|c|c|c|}
\hline MUTANT & NETA & MUTANT & NET $\Delta$ \\
\hline 1) $\mathrm{D} 16 / \mathrm{DPDPD}$ & +4 & 11) $Y 140 / S R D$ & +2 \\
\hline 2) P44/PLRIR & +4 & 12) G153/GPG & +2 \\
\hline 3) F67/RDPV & +3 & 13) $Y 168 / S R D$ & +2 \\
\hline 4) $D 81 / D Y$ & +1 & 14) K180-K184/DP & -3 \\
\hline 5) S82ISLRIR & +4 & 15) G209/AGSR & +3 \\
\hline 6) $Y 91 / Y R D P D$ & +4 & 16) $A 218 / A D$ & +1 \\
\hline 7) $Y 91 / S D P$ & +2 & 17) A230/ASGSA & +4 \\
\hline 8) $\mathrm{A} 110 / \mathrm{GIP}$ & +2 & 18) H253/RDP & +2 \\
\hline 9) O127/HGSE & +3 & 19) A259/ARA & +2 \\
\hline 10) Q131/HGSE & +3 & 20) A260/GIP & +2 \\
\hline
\end{tabular}

B

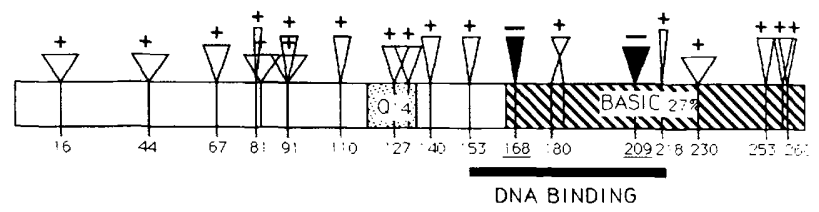

Figure 3. Codon insertion mutagenesis of HAP2. (A) Summary of the 20 codon insertion mutations constructed as described in Materials and methods. The sequence changes made are indicated by the HAP2 amino acids and codon numbers destroyed, followed by a slash and the amino acids inserted at that site by linker sequence and/or Klenow fill-in. The net addition for deletion) of codons is also indicated. ( $B$ ) Summary of the position, size, and phenotype of the 20 codon insertion mutations. The structure of HAP2 is represented schematically, and the positions of the polyglutamine tract (Q14, stippled) and carboxy-terminal basic region (hatched) are indicated. The minimal region of HAP2 required for assembly and CCAAT DNA binding of the HAP2/3/4 complex (amino acids 154-218) is indicated below. The net insertion of codons is proportional to the size of the base of the inverted triangle. Complementation of a hap2 null mutant for growth on lactate is indicated by plus $|+|$ for wild-type levels of complementation and minus $(-)$ for no detectable complementation.

ably affect lactate growth. Further deletions that impinge on the DNA-binding domain (mutant $\mathrm{l} x \Delta \mathrm{C} 208$ ) abolished complementation. The presence of the bacterial DNA-binding protein lexA in the HAP2 deletion constructs is unlikely to have affected these observations because deletions of HAP2 fused to the bacterial enzyme $\beta$-galactosidase gave similar results /data not shown).

To demonstrate that there is no redundancy in function of the first 154 and last 47 amino acids, mutants with both regions deleted were constructed. Figure 4C shows that residues 154-218 expressed as a fusion to lexA were entirely sufficient to support growth on lactate (mutant $\mathrm{l} x \Delta \mathrm{N} 154 \Delta \mathrm{C} 218$ ). We conclude that residues 154-218 constitute an essential core of the HAP2 protein. This essential core provides all the protein structure necessary for assembly of HAP2 into the HAP2/3/4 heterotrimer, site-specific DNA binding of the resulting complex, and subsequent transcriptional activation of the promoter-bound complex as judged by in vivo complementation for growth on lactate.
The essential core is divisible into subunit association and DNA recognition domains

An assay for subunit interaction in the HAP2/3/4 complex The ability of a lexA-HAP2 fusion to activate at the lexA site is an assay for assembly of the HAP complex, because HAP4 must be incorporated into the active complex. As suggested earlier (Forsburg and Guarente 1989a) and shown below, HAP4 contains an acidic domain that activates transcription. To dissect sequences in the HAP2 essential core required for interaction with other subunits (HAP3 and/or HAP4) from sequences that may be involved in site-specific DNA interactions in the intact complex, we therefore examined in greater detail the properties of a lexA-HAP2 fusion protein that contains HAP2 codons 16-265 fused to the DNA-binding domain of lexA (codons 1-87). Figure 5 shows that the lexA-HAP2 fusion bound at the lexA operator activated transcription, as assayed by a CYC1-lacZ fusion, in the same carbon source and heme-dependent manner that occurs at UAS2. The levels of $\beta$-galactosidase were derepressed 3-fold in galactose and 13-fold in lactate over levels observed in glucose. Similarly, low heme conditions repressed the levels by 11 -fold. These regulatory effects at the lexA site paralleled those observed at UAS2UP1 with wild-type HAP2 or with the bifunctional lexA-HAP2 (not shown). To demonstrate that the effects at the lexA operator were specific, a lexA-GAL4 fusion was also checked and was neither strongly affected by intracellular heme levels nor carbon source (Fig. 5). In contrast to the behavior of lexA-HAP2, the activity of lexA-GAL4 actually decreased in lactate. This reduction in activity is likely due to a decrease in expression of the lexA-GAL4 fusion from the ADH1 promoter, which is reportedly repressed by growth on nonfermentable carbon sources (Denis et al. 1983).

The critical test of the lexA-HAP2/HAP3/HAP4 interaction assay is that activity at the lexA operator should depend on HAP4 and, possibly, HAP3. The roles of the other two subunits of the HAP2/3/4 transcriptional activator were investigated by examining the function of lexA-HAP2 in strains mutant for either HAP3 or HAP4. Although both HAP3 and HAP4 are required for binding to UAS2, activation by lexA-HAP2 at the lexA operator is independent of the ability to bind UAS2. The effect of removing either HAP3 or HAP4 thus directly reflects the role of the protein in transcriptional activation. As shown in Figure 5, a hap3 null mutation resulted in a twofold decrease in transcriptional activation in glucose and an eightfold decrease in galactose. Levels of activation in lactate could not be tested because of the inability of the hap 3 mutant strain to grow on a nonfermentable carbon source. Nonetheless, the decrease in expression in glucose and lack of derepression in galactose strongly suggests that the lexAHAP2 fusion is dependent on the HAP3 subunit for the formation of a strong, regulatable transcriptional activation complex at the lexA operator. Similarly, the activity of lexA-HAP2 in a hap4-2 mutant background failed to show galactose-derepressible transcriptional activation at the lexA operator. Although the effect was 


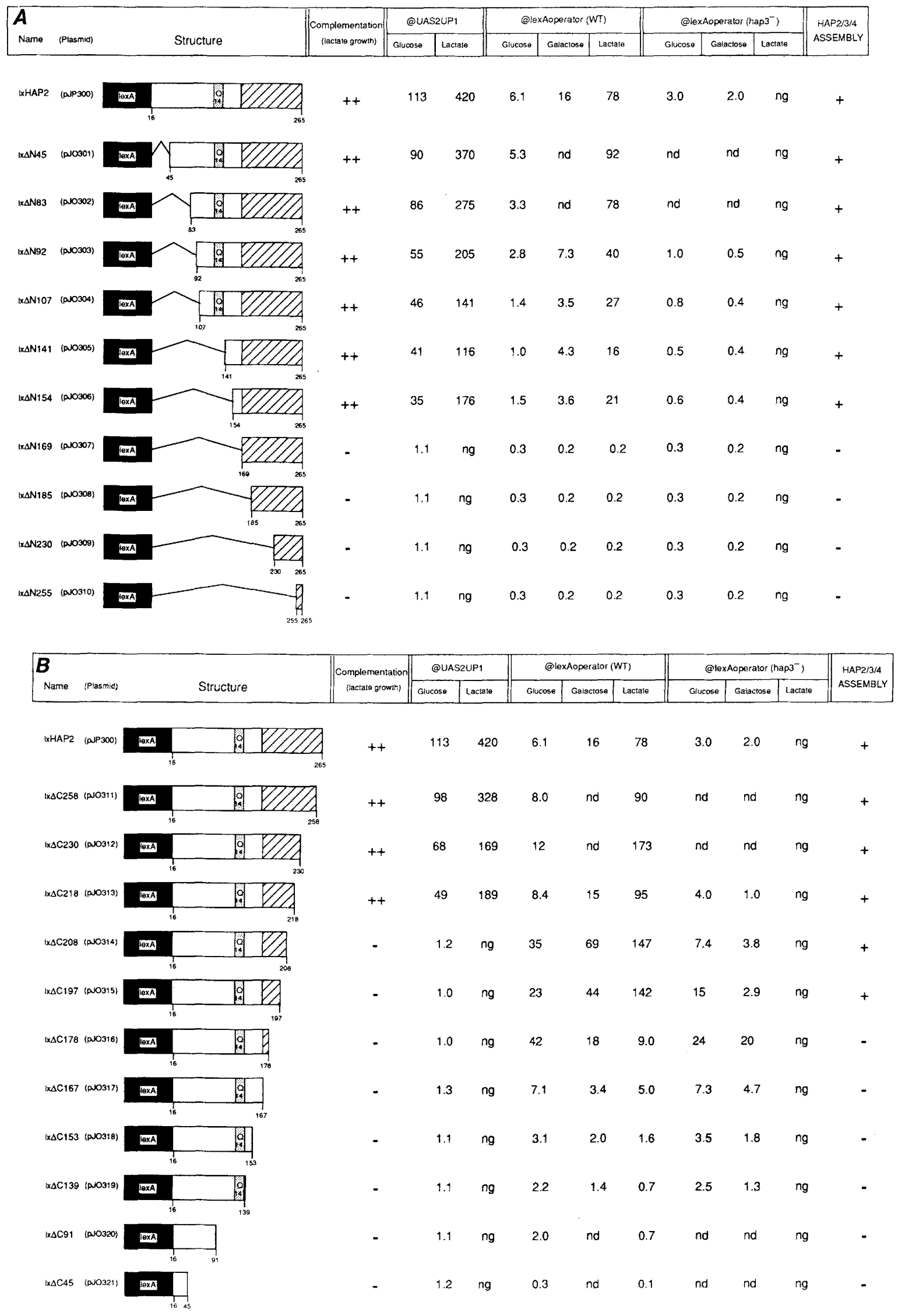

Figure 4. (Continued on next page.) 


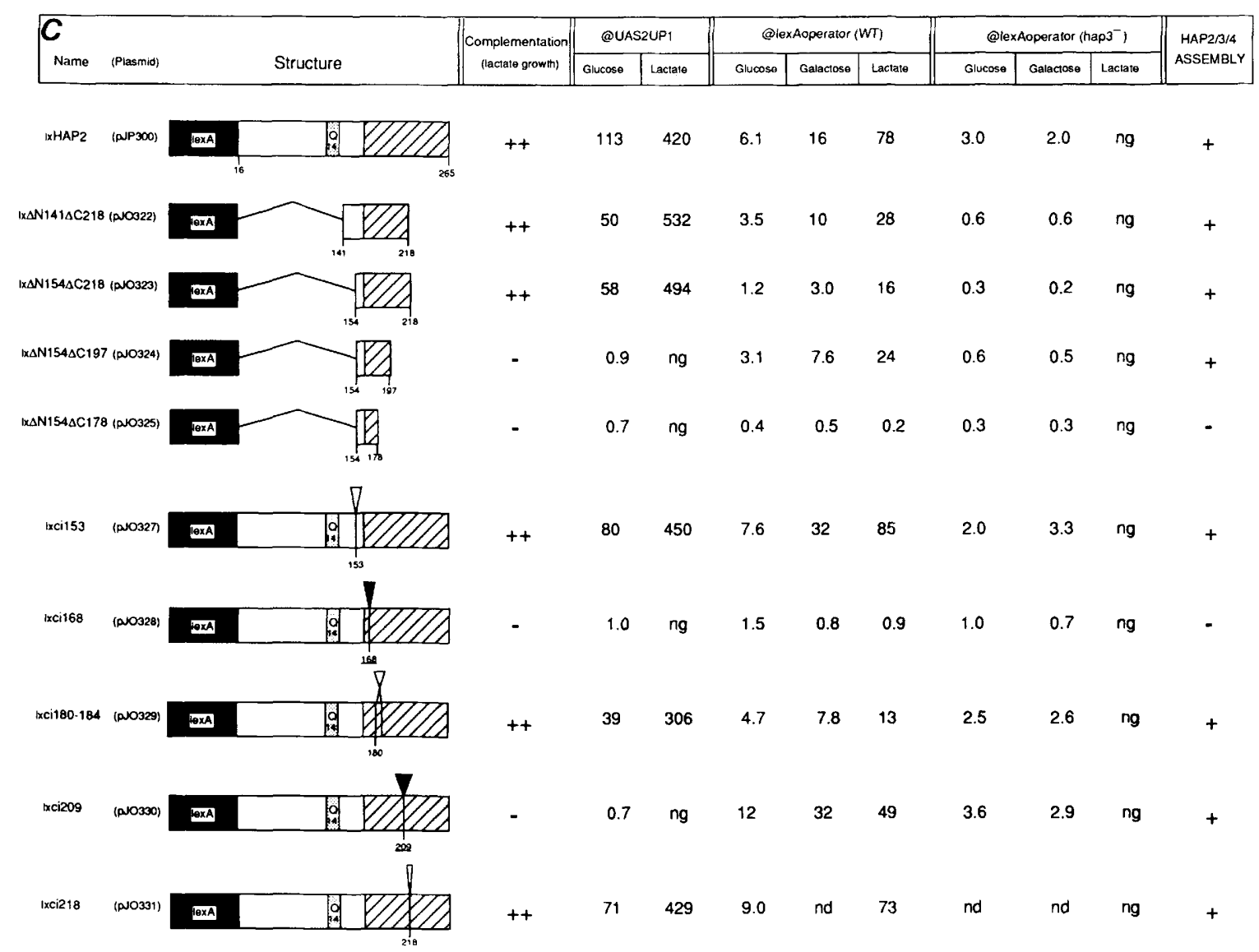

Figure 4. Analysis of transcriptional activation and HAP3/HAP4 subunit assembly by lexA- $\mathrm{HAP} 2$. The structure of the original lexA-HAP2 fusion (lxHAP2) and deletion derivatives thereof are shown schematically with the positions of the polyglutamine tract (Q14, stippled) and carboxy-terminal basic region (hatched) indicated. Numbers represent the first HAP2 codon present in amino-terminal deletions and the last HAP2 codon present in carboxy-terminal deletions. Codons introduced during the construction by added linker sequences are summarized in Materials and methods in the case of deletion constructs and in Fig. 3A in the case of codon insertion constructs. Complementation of a hap2 null mutation is indicated for growth on lactate by the indicated lexA- $\mathrm{HAP} 2$ fusion: $1++\mid$ wild-type complementation; $|-|$ no complementation detected. The data represent the average of at least four independent determinations of units of $\beta$-galactosidase from UAS2UP1 (pLG $265 \mathrm{UP1}$ ) in hap2 null strain (first two columns of numbers) or from the lexA operator (PRB 1155) in a wild-type (WT) yeast strain (next three columns) or in a hap 3 null strain (last three columns of numbers) and the indicated carbon source. The final column indicates whether the given lexA- $\mathrm{HAP} 2$ fusion is assembling with HAP3/HAP4 and is based on the difference between the wild-type and hap3 null strains for levels of activation in glucose and galactose at the lexA operator. Constructs that require the presence of HAP3 for significant levels of activation are judged to be assembling into a lexA- $\Delta$ HAP2/3/4 complex at the lexA operator, whereas those that do not (e.g., $1 \mathrm{x} \Delta \mathrm{C} 178$ and $1 \mathrm{x} \Delta \mathrm{Cl} 167)$ are judged to be activating transcription at the lexA operator independently of the other subunits. $(+$ and -$)$ The given lexA- $\triangle \mathrm{HAP} 3$ construct assembles with HAP3/HAP4 or does not, respectively. $(A)$ Amino-terminal deletions of lexA-HAP2; $(B)$ carboxy-terminal deletions of lexA-HAP2,$(C)$ Double (amino- and carboxy-terminal) deletions of lexA-HAP2, and codon insertion lexA-HAP2 constructs.

less marked than in the hap 3 background, this is likely due to the use of an ethylmethane sulfonate (EMS)-derived point mutant (hap4-2) that is somewhat leaky, rather than a null mutant as was used for the hap 3 experiment. Control experiments with the lexA-GAL4 fusion showed that neither hap 3 nor hap 4 mutant backgrounds nonspecifically affected transcriptional activation through the lexA operator (Fig. 5, and results not shown). We conclude that the HAP2 subunit is capable of assembling in solution with HAP3 and HAP4, and that the activity of lexA-HAP2 at the lexA site is a measure of this assembly. HAP2 in the absence of HAP3 or HAP4 behaves as a weak constitutive transcriptional activator. These observations, which imply the formation of a stable HAP2/3/4 heterotrimer in solution, are consistent with the observation that all three subunits copurify through four chromatographic steps in the absence of DNA (Hahn and Guarente 1988).

The HAP2 essential core contains two domains-one sufficient for subunit association and the other required specifically for CCAAT DNA recognition To map the regions of HAP2 required for association with the HAP3 and HAP4 subunits, amino- and carboxy-terminal deletions of lexA-HAP2 were examined for HAP3-dependent activation at UAS2UP1 and at the lexA operator. As summarized in Figure 4A, amino-terminal deletions extending to amino acid 154 still responded to carbon 


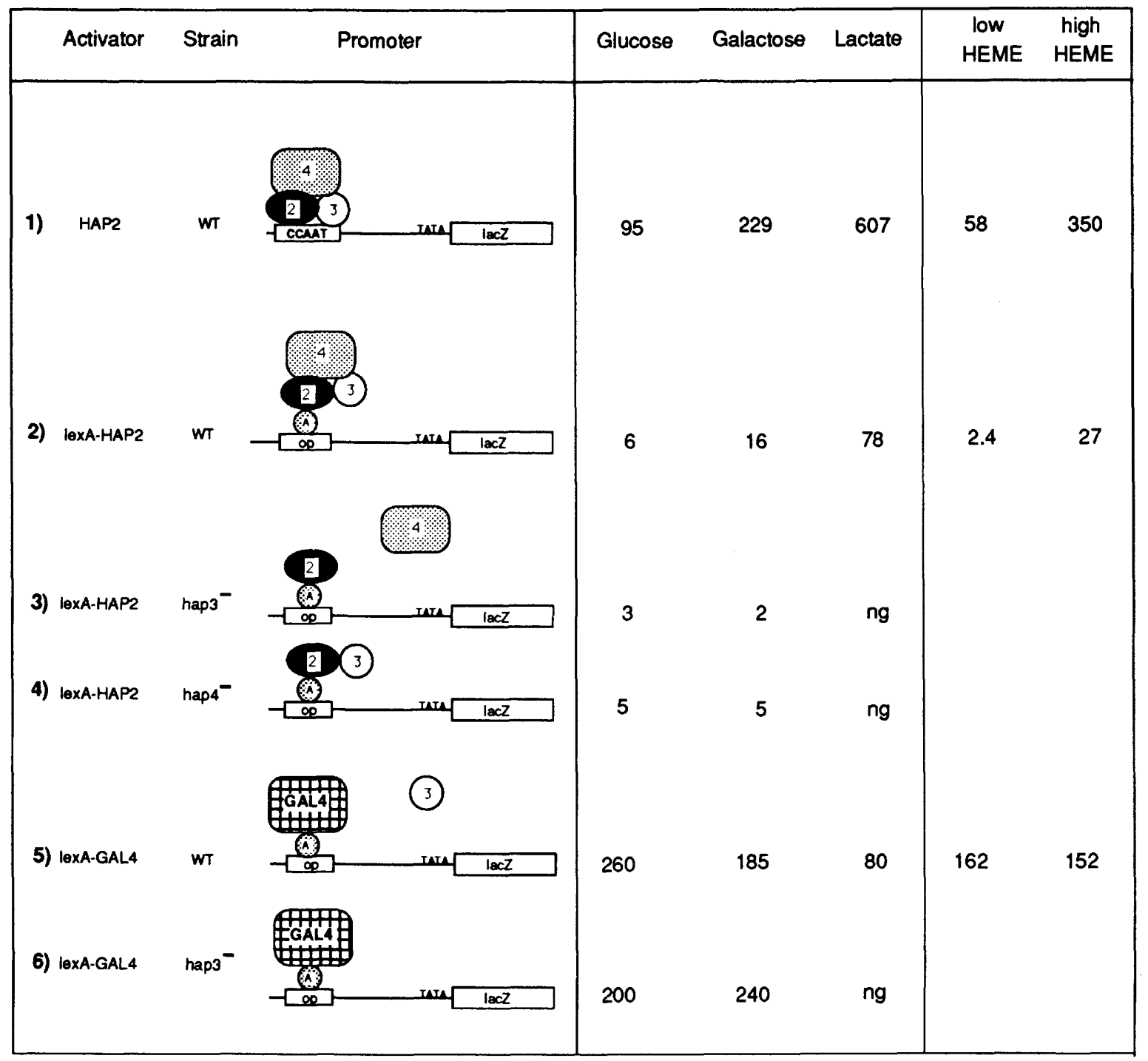

Figure 5. Transcriptional activation by HAP2/3/4 at UAS2UP1, and by lexA-HAP2/3/4 and lexA-GAL4 at the lexA operator. Numbers indicate the average of a least four independent determinations of units of $\beta$-galactosidase activity detected from the indicated $l a c Z$ promoter fusion under the indicated carbon source and heme conditions. The putative configuration of activator proteins in each yeast strain is also indicated. For the sake of simplicity, a single lexA-fusion protein is shown binding to the lexA operator (the lexA repressor actually binds its operator as a dimer), and a single copy of each HAP subunit in the complex is also assumed. HAP2 (black oval), HAP3 (open circle), and HAP4 (stippled form) are shown assembled and bound at the UAS2UP1 CCAAT box (line 1) or the lexA operator via the lexA-HAP2 fusion (line 2). The complex is shown incompletely assembled in either a hap 3 null strain (line 3) or a hap4 null strain (line 4) (see Discussion). Activation by lexA-GAL4 fusion at the lexA operator (lines 5 and 6) is also summarized. This fusion is unaffected by the absence of HAP3 (line 6) or HAP4 (not shown).

source by derepressing strongly in lactate and were HAP3-dependent. Although overall levels of activation decreased as sequences were deleted, no effect was observed on the degree of carbon source regulation or HAP3 dependence. Interestingly, no effect was observed from deletion of the polyglutamine tract $(\mathrm{cf}$. $\mathrm{l} x \Delta \mathrm{N} 107$ with $1 \mathrm{x} \Delta \mathrm{N} 141)$. Further deletions $(\mathrm{l} \Delta \Delta \mathrm{N} 169)$ impinging on the essential core of HAP2 resulted in the complete loss of activation at UAS2UP1 and the lexA operator in all carbon sources, with or without HAP3. We conclude that, although the amino terminus of HAP2 may play a minor role in augmenting transcription of the heterotrimeric complex, it is not involved in assembly of HAP2 with the HAP3 and HAP4 subunits into a regulatable transcriptional activating complex.

The results of carboxy-terminal mutagenesis of lexAHAP2 are summarized in Figure 4B. Deletions extending to amino acid $218(\mathrm{x} \Delta \mathrm{C} 218)$ did not significantly alter gross levels of activation, carbon source regulation, or HAP3 dependence at UAS2UP1 or the lexA operator. The region extending from amino acid 218 to 265 thus appears entirely dispensable for HAP2 function.

Further carboxy-terminal deletions $(1 \mathrm{x} \Delta \mathrm{C} 208$ and $\mathrm{l} \Delta \mathrm{C} 197)$ extending into the essential core of HAP2 are critical in that they indicate that HAP2 has distinct regions dedicated to UAS2 binding and subunit interac- 
tion. These deletions abolished complementation and activation at UAS2 but did not affect carbon source regulation or HAP3 dependence at the lexA operator. We conclude that this region (amino acid 197-218) is essential for site-specific DNA binding but is not required for assembly with the HAP3 and HAP4 subunits to form a regulatable transcriptional activation complex. Further carboxy-terminal deletions ( $\mathrm{x} \Delta \mathrm{C} 178$ and $\mathrm{lx} \Delta \mathrm{Cl}$ 167) obliterated carbon source regulation and HAP3 dependence at the lexA operator. These mutants showed low constitutive levels of activation at the lexA site, consistent with the proposed function of the amino terminus of HAP2 as a transcription-augmenting domain.

Combined amino- and carboxy-terminal deletions of lexA-HAP2 were also examined for their activity at the lexA operator. As summarized in Figure $4 C$, two essential core-encompassing fusions, $\operatorname{lx} \Delta \mathrm{N} 141 \Delta \mathrm{C} 218$ and $\mathrm{l} x \Delta \mathrm{N} 154 \Delta \mathrm{C} 218$, showed normal carbon source regulation and HAP3 dependence at both UAS2UP1 and the lexA operator. Furthermore, deletion $1 \times \Delta N 154 \Delta C 197$, which removes the region required for site-specific DNA binding, functioned normally at the lexA operator but not at UAS2, whereas deletion of additional sequences extending into the putative subunit recognition domain eliminated this activity ( $\mathrm{x} \Delta \mathrm{N} 154 \Delta \mathrm{C} 178)$. We conclude that amino acids 154-197 of HAP2 are both necessary and sufficient for association with HAP3 and HAP4.

To examine further the specificity of the proposed subunit association and DNA-contacting domains of the essential core, we constructed lexA fusion derivatives of several of the codon insertion mutants described above (Fig. 4C). Codon insertion mutants 13 (Y168/SRD) and 15 (G209/AGSR) occur in the subunit association and DNA-contacting domains, respectively. As would be expected for a mutant that specifically disrupts CCAAT binding, lxci209 was inactive at UAS2 but showed normal carbon source regulation and $H A P 3$ dependence at the lexA operator. In contrast, mutant lxcil68 was neither carbon source-regulated nor HAP3 dependent at the lexA operator, as would be expected for a mutant that disrupts assembly with the HAP3 and HAP4 subunits. Mutant lxcil80-184 (K180-K184/DP), which fully complements for growth on lactate, showed slightly compromised levels of activation at the lexA operator but was nonetheless HAP3-dependent, consistent with a slight impairment in the ability to assemble with HAP3 and HAP4 in solution. Other codon insertion mutations examined did not affect the behavior of lexAHAP2.

We conclude that amino acids 154-197 are involved specifically in subunit association and amino acids $198-218$ are involved specifically in DNA recognition. Amino acids 180-184 appear relatively dispensable for assembly with HAP3 and HAP4. These results are summarized in Figure 6, which presents a four-domain model for the structure of HAP2.

\section{Roles of HAP3 and HAP4}

It was critical to validate the view that HAP4 contains the principal activation domain for the HAP2/3/4 complex. Accordingly, we constructed a bifunctional lexAHAP4 fusion, containing the DNA-binding domain of the lexA repressor and codons 10-554 of HAP4. In contrast to the behavior of the lexA-HAP2 fusion at the lexA operator, lexA-HAP4 fusion produced from the $\mathrm{ADH} 1$ promoter was extremely active in promoting transcription in all carbon sources and under both heme-limiting and heme-sufficient conditions (Fig. 7A). Furthermore, levels of transcriptional activation actually increased slightly in the absence of HAP2 and/or HAP3. These data show that HAP4 encodes a strong transcriptional activation domain.

Is the sole role of HAP4 to bring the activation domain to the complex? Previous work suggested that HAP4 was required for sequence-specific DNA binding, because a gel shift complex from hap 4 mutant cells was not observed (Forsburg and Guarente 1989a). However, we do not believe that this binding assay is sensitive. We therefore employed a genetic scheme to examine whether HAP4 was absolutely required for UAS2-specific DNA binding in the complex. If the only role of the HAP4 subunit is to provide a transcriptional activation domain to the $\mathrm{HAP} 2 / 3 / 4$ heterotrimer, then we reasoned that the need for HAP4 could be bypassed by merely fusing an acidic transcriptional activation domain to one of the other two subunits.

As shown in Figure 7B, fusing the 129-amino-acid GAL4 acidic tail to the carboxyl terminus of HAP2 results in a partial bypass of the need for HAP4. The HAP2-GAL4 acid blob fusion supported partial growth on lactate in hap4 null background. Thus, HAP2 and HAP3 alone will bind to the CCAAT sites in the yeast genome. The suboptimal level of complementation may be because HAP4 has a quantitative effect on binding of the HAP complex to its sites. Alternatively, it may be due to repression of the GAL4 activation domain by GAL80, by instability of the HAP2-GAL4 fusion protein, or an unfavorable effect of the acidic activation domain on site-specific DNA binding by HAP2.

Notably, the HAP2-GAL4 acid blob fusion did not eliminate the need for HAP3. This finding indicates that the role of HAP3 in the complex is not merely to serve as a connector between HAP2 and HAP4. Rather, the HAP3 subunit must be essential for site-specific DNA binding. Thus, the subunits of the HAP2/3/4 hetero-

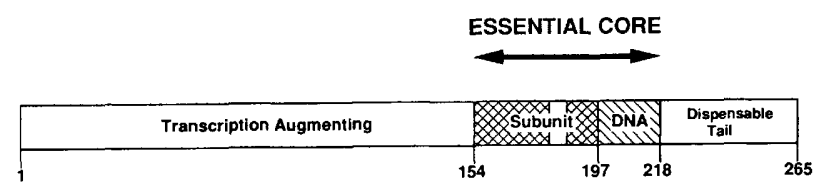

Figure 6. Four-domain model for the structure of HAP2. The essential core of HAP2 (amino acids 154-218) is shown encompassing both subunit association (crosshatched) and DNA recognition (hatched) domains. The gap occurring in the subunit association domain is meant to represent the nonessential sequences (amino acids 180-184) occurring in this region (see codon insertion mutagenesis). The two nonessential domainsthe amino-terminal transcription-augmenting and the carboxyterminal-dispensable tail region-are also indicated. 

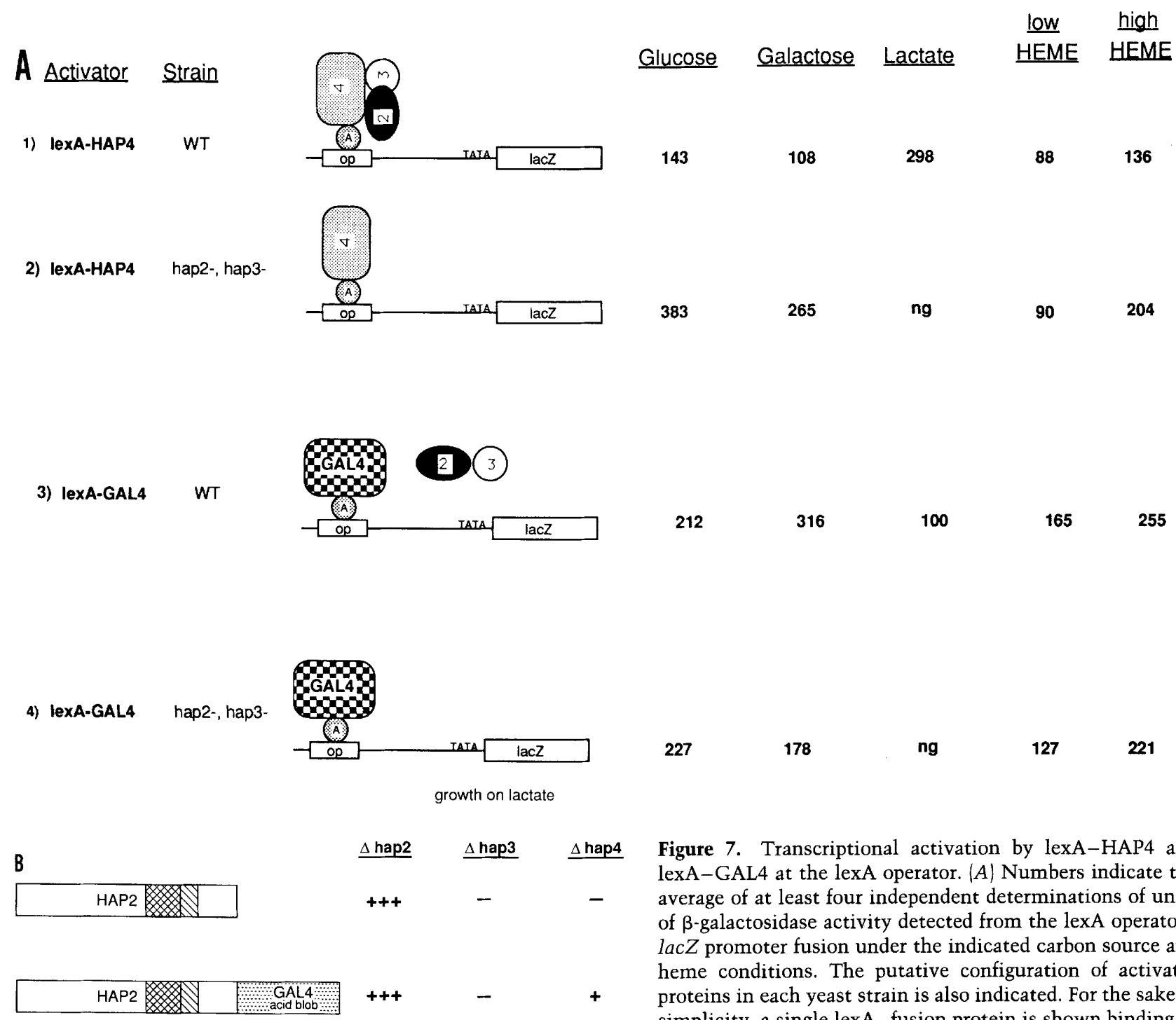

Figure 7. Transcriptional activation by lexA-HAP4 and lexA-GAL4 at the lexA operator. $\{A\}$ Numbers indicate the average of at least four independent determinations of units of $\beta$-galactosidase activity detected from the lexA operator$l a c Z$ promoter fusion under the indicated carbon source and heme conditions. The putative configuration of activator proteins in each yeast strain is also indicated. For the sake of simplicity, a single lexA-fusion protein is shown binding to the lexA operator (the lexA repressor actually binds its operator as a dimer), and a single copy of each HAP subunit in the complex is also assumed. HAP2 (black oval), HAP3 (open circle) and HAP4 (stippled form) are shown. Essentially identical results were obtained for lexA-HAP4 in either the hap2/hap3 null strain (line 2 ) or individual hap2 null and hap3 null strains (data not shown). (B) A HAP2-GAL4 acidic domain fusion partially bypasses the need for HAP4. The structures of HAP2 and the HAP2-GAL4 fusion containing the acidic carboxy-terminus of GAL4 are shown schematically. Complementation for growth on lactate is shown in each of the three hap null strains. $(+++\mid$ Fully wild-type levels of lactate growth; $|+|$ slow but significant growth on lactate. $|-|$ No detectable complementation of the lactate growth defect phenotype.

trimer can be crudely divided into DNA binding (HAP2 and $\mathrm{HAP} 3$ ) and transcriptional activation (HAP4) functions. These results are summarized in Figure 8 , which presents a functional model for the HAP2/3/4 complex.

\section{Discussion}

In most cases of well-examined eukaryotic transcriptional activators, a single polypeptide has been shown to encode small discrete domains for both DNA binding and transcriptional activation. Increasingly, complex transcriptional activators consisting of two or more different polypeptides have been recognized and characterized. Currently, this class of regulatory proteins includes yeast HAP2/3/4 and its apparent mammalian homolog CP1 (Olesen et al. 1987; Chodosh et al. 1988a,b), leucine zipper-containing proteins such as mammalian fos/jun and other AP-1-related complexes (for review, see Curran and Franza 1988), as well as the activating transcription factor (ATF) family of transcription factors (Hai et al. 1989). MyoD and other proteins containing the helix-loop-helix dimerization/DNA-binding motif (Murre et al. 1989a) have also been shown to form DNAbinding heterodimers (Murre et al. 1989b). The discovery of an ever-increasing number of complex transcriptional regulators involved in the control of gene expression in higher cells may have profound implications for understanding metazoan gene regulation through combina- 
torial control mechanisms. A thorough understanding of the structure and function of this class of regulators not only requires a knowledge of the subunit protein domains involved in DNA binding and transcriptional activation but also the protein sequences involved in heteromeric protein-protein interactions. In this study, we begin a functional dissection of the HAP2/3/4 CCAAT-binding complex by investigating the general roles of the HAP2, HAP3, and HAP4 subunits in transcriptional activation and DNA binding. In addition, we begin a molecular dissection of the complex by localizing a region of HAP2 responsible for interaction with HAP3 and possibly HAP4, and a region of HAP2 specifically involved in DNA recognition.

\section{Structure of HAP2}

Using deletion mutagenesis and an in vitro DNAbinding assay, we demonstrated that amino acids 154-218 of HAP2 provide all of the necessary information for assembly of HAP2 into a CCAAT-binding complex. This region of HAP2 was necessary and sufficient for cooperative binding with both $S$. cerevisiae HAP3/ HAP4 and with mammalian CP1A, the functional HeLa homolog of these yeast activities (Chodosh et al. 1988b). Deletions of HAP2 had identical effects on cooperative

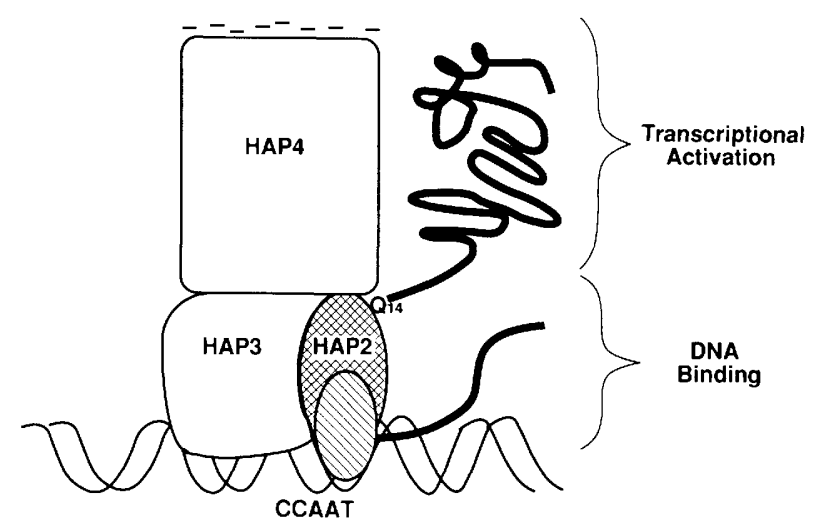

Figure 8. Proposed structure of the HAP $2 / 3 / 4$ CCAATbinding complex and division of site-specific DNA binding and transcriptional activation functions between the subunits. The model hypothetically assumes a single copy of each of the three subunits in the heterotrimeric complex. The detailed structure of HAP2 is shown, with its essential core (ovals) consisting of subunit association (crosshatched oval touching HAP3) and DNA recognition (hatched oval touching the DNA double helix) domains. The putative transcription-augmenting amino terminus is represented by a random wavy line connected to the subunit association domain via the polyglutamine tract (Q14). The carboxy-terminal dispensable tail is also indicated. HAP3 and HAP4 subunits are depicted as uncharacterized shapes. HAP3 is shown contacting the DNA, HAP2, and HAP4. In contrast, HAP4 is presumed not to contact the DNA and is shown making protein-protein contacts with HAP3 and, possibly, HAP2. The HAP4 acidic transcriptional activation domain is represented by the negative charges at the top of that subunit. binding with yeast HAP3/HAP4 and HeLa CP1A, thus providing further evidence that the yeast and mammalian CCAAT-binding protein complexes have been conserved extensively in both structure and function throughout the period of evolutionary divergence between yeast and man. In other work (J. Olesen and $\mathrm{L}$. Guarente, unpubl.), we present evolutionary evidence that the HAP2 core is a highly constrained protein structure that has changed in amino acid sequence very little since the divergence of budding yeast, fission yeast, and metazoan eukaryotic cell lineages. By use of codon insertion and deletion mutagenesis of HAP2, we have demonstrated that this core region of HAP2 is entirely sufficient for functional complementation in vivo. HAP2 residues $154-218$ thus constitute an essential core domain of this protein.

The observation of carbon source-regulated, heme-dependent, and HAP3/HAP4-dependent activation by lexA-HAP2 tethered to a yeast promoter via the lexA operator provides an in vivo assay for the association of HAP2 with the other subunits in the complex. Using this assay, we localized a small region of the HAP2 protein that is both necessary and sufficient for association with HAP3 and HAP4. The subunit association domain is a subset of the essential core of HAP2, which encodes both subunit association and DNA-recognition activities. HAP2 residues $154-197$ span the subunit association domain because they are sufficient for the assembly of the HAP2/3/4 complex in solution at the lexA operator. A carboxy-terminal deletion to residue 178 destroyed subunit association with HAP3/HAP4 (see Fig. $4 \mathrm{~B}, \mathrm{x} \Delta \mathrm{C} 178$, and Fig. $4 \mathrm{C}$, $\mathrm{x} \Delta \mathrm{N} 154 \Delta \mathrm{C} 178)$, whereas a mutation substituting codons $180-184$ with two other codons resulted in only a slight impairment of subunit association as measured at the lexA operator. This suggests that residues $180-184$ are somewhat dispensable and that either the precise spacing between residues 154 and 178 and 185 and 197 is also not critical for the function of the subunit association domain, or that the latter region is also dispensable and the minimal subunit association domain extends only to amino acid 179 .

The remainder of the HAP2 essential core /amino acids $197-218$ ) is clearly critical for site-specific DNA binding because it is required for activity at the UAS2UP1 CCAAT box. The clear implication from these results is that site-specific DNA binding is determined by these HAP2 residues, along with the other subunits. The DNA-binding domain of HAP2 thus constitutes the entire essential core structure, including the subunit association domain. This arrangement is similar to that occurring in the DNA-binding domain of FOS (Neuberg et al. 1989), Jun, and related leucine zipper and helix-loop-helix proteins. Indeed, the HAP2 subunit association and DNA-recognition domains apparently constitute novel, previously undescribed structures for heteromeric protein/protein interaction and site-specific DNA binding because they show no homology to previously described DNA-binding motifs, the leucine zipper (Landschulz et al. 1988), helix-loop-helix (Murre et al. 1989a), helix-turn-helix (Brennan and Matthews 
1989), homeo domain (Scott et al. 1989), or zinc finger (Klug and Rhodes 1987).

Whereas residues $219-265$ comprise a dispensable tail region of HAP2, the amino-terminal 154 residues appear to have subtle effects on transcriptional activation of the assembled complex assayed at the lexA operator. Gross levels of activation, but not the degree of carbon source regulation, at UAS2UP1 and the lexA operator diminished as sequences 16-154 were deleted ( $1 \mathrm{x} \Delta \mathrm{N} 45-$ $\mathrm{l} \Delta \Delta$ N154, Fig. 4A). Significantly, however, deletion of the polyglutamine motif had no effect on transcriptional activation at the lexA operator or UAS2. The amino terminus of HAP2 on its own functioned as a weak constitutive transcriptional activator at the lexA operator (see Fig. 4B, $1 \mathrm{x} \Delta \mathrm{C} 178-1 \mathrm{x} \Delta \mathrm{C} 139$ ). In contrast, the dispensable tail region had no such activity $(\mathrm{l} x \Delta \mathrm{N} 169-\mathrm{l} x \Delta \mathrm{N} 255$, Fig. 4A). The calculated net charge of this putative transcriptional augmenting domain is -10 which, although not as densely acidic as previously identified activating regions (Ma and Ptashne 1987a), is consistent with a minor role in transcriptional activation of the assembled HAP2/3/4 complex.

\section{Division of functions among the subunits}

In addition to mapping subunit association and DNArecognition domains of HAP2, we presented evidence for a division of function between the subunits of the heterotrimer. The nature of the HAP2/3/4 complex requires that all three subunits encode surfaces for protein-protein interaction between the subunits that allow assembly of the complex. In contrast, each of the remaining functions of a transcriptional activator, that is, site-specific DNA binding and transcriptional activation, need not be specified by all of the subunits of the complex. Indeed, the experiments presented here suggest that HAP2 and HAP3 appear to provide the specificity for CCAAT DNA binding, whereas HAP4 appears to provide the primary transcriptional activation surface to the HAP2/3/4 complex (Fig. 8). The evidence justifying this division of function among the subunits is as follows.

HAP4 encodes the principle transcriptional activation domain of the complex It has been demonstrated previously that HAP4 contains an essential and highly acidic protein domain at its carboxyl terminus (Forsburg and Guarente 1989a). This domain can be exchanged with the acidic activation domain of GAL4 without impairing the function of the CCAAT activation complex, suggesting that the HAP4 acidic sequence plays the same role in transcriptional activation as the acidic sequence of GAL4. We sought independent confirmation that the HAP4 subunit provides the principle transcriptional activation domain for the entire complex by assaying each of the subunits independently for its activation potential at the lexA operator. In the absence of HAP4 neither lexA-HAP2 nor lexA-HAP3 (not shown) is capable of activating transcription at high levels through the lexA operator. This suggests that strong ac- tivation surfaces do not appear on either HAP2 or HAP3. In contrast to lexA-HAP2 and lexA-HAP3 fusions, lexA-HAP4 conferred very strong carbon source and heme-independent transcriptional activation on the lexA operator-driven promoter. Furthermore, the HAP2 and HAP3 subunits were not required for transcriptional activation by lexA-HAP4.

A different approach also indicated that HAP4 brings the activation domain to the complex. Fusion of the acidic activation domain of GAL4 to the carboxyl end of HAP2 partially bypassed a hap4 deletion mutation. This result shows that HAP4 is not absolutely required for DNA site recognition by the complex.

HAP2 and HAP3 encode the CCAAT DNA-binding specificity The HAP2-GAL4 fusion that partially bypassed the need for HAP4 clearly did not bypass the need for HAP3. Because HAP3 is not needed for the nuclear localization of HAP2 (Pinkham et al. 1987), this result strongly suggests that HAP3 is required for HAP2 to recognize the CCAAT box sequence. Furthermore, a protein fusion of HAP2 to HAP4, while fully complementing both hap2 and hap4 null mutations, does not bypass the need for HAP3, as might be expected if HAP3 functioned only to link the two subunits together. (J.T. Olesen and L. Guarente, unpubl.). An additional argument for a role of HAP3 in site-specific DNA binding is the UAS2 element itself is bipartite and asymmetric (Forsburg and Guarente 1988). Binding to the asymmetric UAS2 might involve an asymmetric arrangement of dissimilar subunits (i.e., HAP2 and HAP3) of the complex.

\section{Regulation by the HAP2/3/4 complex}

The static model for the HAP2/3/4 complex does not explain how carbon source and heme regulation of UAS2 occurs. In the case of another complex transcriptional activator, mammalian Fos-Jun heterodimer, transcriptional regulation through the Fos-Jun-binding site (TRE) occurs in response to serum stimulation via changes in the amount of Fos protein synthesized.

The data presented here, in combination with existing evidence, suggests that transcriptional regulation through the UAS2UP1 CCAAT box by HAP2/3/4 occurs via a similar modulation of the amount of assembled heteromeric complex. In this case, it is the HAP4 subunit that appears to play the rate-limiting role served by Fos in the Fos-Jun complex. The evidence for this is as follows. First, measurable DNA-binding activity by HAP2/3/4 requires the presence of all three subunits of the complex and is strongly induced by carbon source (Olesen et al. 1987; Forsburg and Guarente 1989a). Furthermore, both the HAP3 and HAP4 subunits are required for induction by lexA-HAP2 at the lexA operator. Together, these facts suggest that induction of the HAP2/3/4 DNA-binding activity occurs by assembly of the HAP2/3/4 complex in solution. Second, evidence that the HAP4 subunit is synthesized in limiting amounts under repressing conditions comes from anal- 
ysis of HAP4 gene expression (Forsburg and Guarente 1989a). Northern analysis reveals that levels of HAP4 mRNA increase fivefold in a shift from glucose to lactate and similar effects are observed for $\beta$-galactosidase levels from a HAP4-lac $Z$ fusion under control of the HAP4 promoter. Future analysis of the HAP4 promoter may reveal a control element, analogous to the c-fos SRE, which is responsive to carbon source growth conditions.

It is interesting to note that for both the Fos-Jun (Lech et al. 1988) and the proposed HAP2/3/4 regulation scheme, the limiting subunit possesses a potent transcriptional activation domain. Similar strong transcriptional activators have been shown to have an adverse affect on RNA polymerase II-dependent mRNA synthesis when present in high concentrations (for review, see Ptashne 1988). Limiting the synthesis of the most potent transcriptional activating subunit in a heteromeric complex avoids this problem while, at the same time, providing a straightforward mechanism for controlling activation from the heteromeric complex.

Further analysis of the structure and function of the HAP2/3/4 complex will provide important insights into the nature of the subunit association and DNA-recognition motifs utilized by this heteromeric transcriptional regulator. In addition, analysis of the HAP $2 / 3 / 4$ regulatory network will aid our understanding of the elaborate combinatorial control mechanisms affecting eukaryotic gene regulation.

\section{Materials and methods \\ Strains and media}

The S. cerevisiae strain BWG 1-7a (MATaleu2-3, 112 his4-519 ade1.100 ura3-52; Guarente and Mason 1983) or an isogenic derivative was used in all experiments described. HAP2 complementation was assessed by using strain JO1-la, which contains a chromosomal deletion of the HAP2 gene obtained by the two-step gene transplacement technique (Rothstein 1983). The hap3-1 and hap3: HIS4 null strain SHY40 have been described (Hahn et al. 1988). The strain JO2-1 is an isogenic hap2,hap3 : HIS4 double null strain derived from JO1-1a by the disruption of the HAP3 gene, as described previously (Hahn et al. 1988). The hap4-2 and hap4 : LEU2 null strain SLF401 have also been described previously (Forsburg and Guarente 1989a). The hem1 null strain MH1-4c (Haldi and Guarente 1989) was used in the heme induction experiments.

Rich media and synthetic minimal media were supplemented with $2 \%$ glucose, $2 \%$ galactose, or $2 \%$ lactate, as described by Sherman et al. (1986). Low heme synthetic media contained $2 \%$ galactose and $0.1 \mu \mathrm{g} / \mathrm{ml} \delta$-ala heme biosynthetic precursor, whereas high heme synthetic media contained $2 \%$ galactose and $50 \mu \mathrm{g} / \mathrm{ml}$ of $\delta$-ala.

\section{Plasmids}

Vectors pRB1 155 (Brent and Ptashne 1985), pJP300 (Pinkham et al. 1987), and pLGA265 [UAS2(CCAAC)-lacZ; Guarente et al. 1984] and pLG $\Delta 265$ UP1(CCAAT)-lacZ; Guarente et al. 1984] have been described.

\section{$\beta$-Galactosidase assays}

The appropriate isogenic yeast strain was transformed with the indicated lacZ reporter $\left(2 \mu, U R A 3^{+}\right)$and lexA fusion effector plasmids $\left(2 \mu, L E U 2^{+}\right)$by sequential transformation, using the lithium acetate protocol (Sherman et al. 1986). Transformants were grown selectively in minimal media (salts plus glucose/ uracil/leucine) and assayed for $\beta$-galactosidase activity as described previously (Guarente and Mason 1983). Values reported were normalized to a control and represent the average of not less than four independent determinations.

\section{Codon insertion mutagenesis}

DNA isolation and manipulation techniques were, in general, as described in Sambrook et al. (1989). HAP2 codon insertion mutants were constructed by use of vector pSP64: HAP2, which contains a 1.4-kb MnlI-EcoRI fragment encompassing the entire HAP2 ORF, as well as 22 bp $5^{\prime}$ and 600 bp $3^{\prime}$ cloned into the SmaI-EcoRI backbone of pSP64 (Promega Biotech). pSP64 : HAP2 was linearized by total or partial digestion with the following enzymes for the indicated codon insertion construct: BgIII (1), StyI (2), MboII (3), HinfI (4), StyI (5), AccI (6 and 7), PstI (8), Fnu4HI (9 and 10), RsaI (11), NlaIV (12), ScaI (13), AluI (14), MboII (15), DdeI (16), NheI (17), SphI (18), PvaII (19), and PstI (20). Full-length linearized vectors were gel-purified by standard techniques in the case of constructs utilizing partial digests before further manipulation to assure cutting at only one site in the plasmid. Linearized ends were rendered blunt with Klenow fragment (Boehringer Mannheim), resulting in a net insertion at $5^{\prime}$ ends ( $1 \mathrm{bp}$, mutants 9 and 10; $2 \mathrm{bp}$, mutants 6 and $7 ; 3$ bp, mutants 4 and 16; $4 \mathrm{bp}$, mutants $1,2,5$, and 17) and a net deletion at $3^{\prime}$ ends ( 1 bp, mutants 3 and $15 ; 4 \mathrm{bp}$, mutants 8,18 , and 20 l. Mutant 7 was constructed by chewing back the 2-base 5' AccI site overhang with mung bean nuclease. Adjacent AluI sites resulted in a net deletion of $17 \mathrm{bp}$ in construct 14. The remaining codon insertion mutants were at blunt sites and did not remove HAP2 sequence. Standard phosphorylated linkers (New England Biolabs, NEB) were ligated to the blunted ends, and the plasmid was recircularized with T7 DNA ligase. Linker size was adjusted to retain the reading frame of $H A P 2$ across the insertion site as follows: SmaI 6-mer (mutants 11, $12,13,14$, and 19), BamHI 8-mer (mutants, 1, 2, 5, 7, 9, 10, and 17), BamHI 10-mer (mutants $3,6,8,15,18$, and 20), or no linker (mutants 4 and 16). The resulting pSP64: HAP2 codon insertion constructs were linearized with the linker site enzyme, religated, and retransformed to ensure that only a single linker remained at the insertion site. The sequence changes resulting from these manipulations are listed in Figure 3. These codon insertion mutants were reconstructed from the $1.35-\mathrm{kb} B g I I I-$ EcoRI HAP2 mutant fragment and the BglII-EcoRI backbone of the ars-cen yeast vector pJP103 (Pinkham and Guarente 1985) for analysis of hap2 complementation. The sequences of the reconstructed mutants were confirmed by restriction analysis, and, in the case of all codon insertion mutants yielding a hap2 phenotype and a majority of those yielding a $\mathrm{HAP2} 2^{+}$phenotype, by M13 DNA sequencing using the Sanger method and commercial reagents (Sequensase).

\section{HAP2 DNA-binding analysis}

Analysis of in vitro DNA binding by HAP2 has performed as follows. The vector pSP64 : HAP2 (described above) was linearized with EcoRI, and synthetic HAP2 RNA was prepared with SP6 RNA polymerase and reagents supplied by Promega Biotech under the conditions recommended for the production of 
large amounts of uncapped and unlabeled RNA. Control RNA was prepared from linearized control pSP64 templates supplied by the manufacturer. The resulting RNA was extracted twice with phenol and ethanol precipitated with $2 \mathrm{M} \mathrm{Na}$ acetate. The RNA was translated in vitro by use of a commercially available rabbit reticulocyte lysate (Promega Biotech) under the reaction conditions recommended for the preparation of large amounts of unlabeled protein. The mock translation reactions were prepared by use of the control RNA described above. The use of control RNA translation products or translations in the absence of exogenous synthetic RNA produced the same results as with this mock translation. Small amounts of these reactions were translated in the presence of $\left[{ }^{35} \mathrm{~S}\right]$ methionine (Amersham), and the resulting radiolabeled proteins were analyzed for size in an SDS-PAGE gel basically as described in Pfeifer et al. (1989). DNA binding was assessed either in the absence of HAP2/ HAP3 activities, or in the presence of $10 \mu \mathrm{g}$ of HeLa fraction $\mathrm{AB}$ containing the CP1A activity (Chodosh et al. 1988b) or yeast HAP2/HAP3 activities supplied from $30 \mu \mathrm{g}$ of crude whole-cell extract of yeast strain JO1-1a ( $\triangle$ hap2) prepared as described previously (Olesen et al. 1987). Preparation of UAS2 and UAS2UP1 probes, as well as binding conditions and native polyacrylamide gels for the resolution of protein-DNA complexes, were as described previously (Olesen et al. 1987; Chodosh et al. 1988b).

Amino- and carboxy-terminal deletions of HAP2 were generated by using the above protocol on deleted derivatives of the pSP64 : HAP2 template prepared as follows. $\triangle$ N43 is a BgIIIBamHI deletion of pSP64: HAP2, which makes HAP2 codon 43 the first ATG downstream of the SP6 promoter. $\triangle N 83$ is a StyI-StyI in-frame deletion of $\Delta \mathrm{N} 43$ that deletes amino acids 45-82 and leaves codons 43 and $44(\mathrm{M}, \mathrm{P})$ at the amino terminus of the ORF. $\triangle \mathrm{N} 92$ is a BglII-BamHI deletion of codon insertion 7, which leaves the first 16 amino acids of HAP2 plus a $\mathrm{P}$ codon fused to codon $92 . \Delta \mathrm{N} 107$ was obtained by ligation of blunted BglII and SpeI sites of pSP64: HAP2, which leaves the first 16 amino acids plus a single codon (P) fused to codon 107. $\Delta \mathrm{N} 141$ is a blunted BglII-SmaI deletion of codon insertion 11, which leaves the first 16 amino acids plus two codons (R,D) fused to HAP2 codon 107. $\triangle$ N154is a BgIII-BamHI deletion of a derivative of codon insertion 12, which contains a BamHI 8-mer linker at the introduced SmaI site (see above). $\Delta \mathrm{N} 154$ thus encodes the first 16 amino acids of HAP2 plus two codons $(\mathrm{P}, \mathrm{G})$ fused to residue 154 . $\Delta \mathrm{N} 169$ is a BglII (blunted)-SmaI deletion of 14 , which leaves the first 16 amino acids plus two codons (R,D) fused to HAP2 codon 169. Most of the carboxy-terminal deletion mutants were generated from pSP64: HAP2 truncated at a restriction site internal to the HAP2 ORF as follows: $\Delta \mathrm{C} 258$ (PvuII), $\Delta \mathrm{C} 230$ (NheI), $\Delta \mathrm{N} 154 \Delta \mathrm{C} 218$ (DdeI), $\Delta \mathrm{C} 167$ (ScaI, $\Delta \mathrm{Cl10}$ (PstI). $\Delta \mathrm{C} 197$ was created by Klenow fill-in of a HinfI site in pSP64: HAP2, which creates an ochre stop codon after residue 197 . Double deletions were created by truncation of $\Delta$ N154 as follows: $\Delta \mathrm{C} 258$ (PvuII), $\Delta \mathrm{C} 252$ (SphI, $\Delta$ N 154 $\Delta \mathrm{C} 230$ (NheI), $\Delta \mathrm{C} 218$ (DdeI), $\Delta \mathrm{N} 154 \Delta \mathrm{C} 208$ (MboII), and $\Delta \mathrm{N} 154 \Delta \mathrm{C} 197$ (HinfI). Constructs were checked for the synthesis of the expected size protein as described above, and the sequence of all templates testing negative in the DNA-binding assay as well as most templates testing positive was confirmed by $\mathrm{M} 13$ sequencing.

\section{lexA-HAP2 deletion constructions}

pIP300 has been described (Pinkham et al. 1987). Deletions of HAP2 fused to the first 87 codons of lexA (plasmids pJO301pIO331) were constructed via the intermediate pSP64: lexAHAP2, which contains the $1.7-\mathrm{kb}$ HindIII-HindIII lexA-HAP2 fragment from pJP300 cloned into the HindIII site of pSP64RI*.
pSP64RI ${ }^{*}$ was derived from pSP64 by restriction with EcoRI, Klenow fill-in, and religation to destroy the EcoRI site. Aminoterminal deletions were reconstructed by ligation of the appropriate size BamHI linker (NEB) onto Klenow-blunted ends of pSP64 : HAP2 linearized with the indicated restriction enzyme as described below, for the given construct: StyI + BamHI 12mer (lx $\Delta \mathrm{N} 45)$, StyI + BamHI 12-mer (lx $\Delta \mathrm{N} 83)$, AccI [blunted with mung bean nuclease $(\mathrm{NEB})]+B a m \mathrm{HI}$ 8-mer (lx $\Delta \mathrm{N} 92$, SpeI + BamHI 10-mer (lx $\Delta$ N107), ScaI + BamHI 10-mer

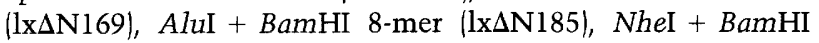
10-mer (lx $\Delta \mathrm{N} 230)$, and SphI + BamHI 10-mer (lx $\Delta \mathrm{N} 255)$. $1 \mathrm{x} \Delta \mathrm{N} 141$ and $\mathrm{lx} \Delta \mathrm{N} 154$ were constructed from codon insertion mutants 11 and 12 by adding a BamHI 10 -mer and a BamHI 8-mer at the SmaI insertion site, respectively. The resulting BamHI-EcoRIDHAP2 restriction fragment was reconstructed as a lexA fusion by ligation into the $B g l I I-E c o R I$ (partial) backbone of pSP64 : lexA-HAP2. The lexA- $\mathrm{HAP} 2$ fusion was released from this intermediate construct on a HindIII-HindIII fragment and inserted into the HindIII site of pAAH5 (Ammerer 1983) for expression in yeast. Insert orientation was screened to ensure transcription of the lexA- $\mathrm{HAP} 2$ construct by the ADHI promoter. Codons introduced at the lexA/ $\triangle H A P 2$ fusion junction by added linker sequences are as follows: $(P, R)$ added in $1 \mathrm{x} \Delta \mathrm{N} 45$ and $\mathrm{lx} \Delta \mathrm{N} 83$; $(\mathrm{P})$ added in $\mathrm{lx} \Delta \mathrm{N} 92, \mathrm{~lx} \Delta \mathrm{N} 185$, and $\mathrm{lx} \Delta \mathrm{N} 230 ;(\mathrm{P}, \mathrm{A})$ added in $\mathrm{lx} \Delta \mathrm{N} 107$ and $\mathrm{lx} \Delta \mathrm{N} 255 ;(\mathrm{P}, \mathrm{G}, \mathrm{D})$ added in $\mathrm{l} \Delta \Delta \mathrm{N} 141 ;(\mathrm{P}, \mathrm{G})$ added in $\mathrm{lx} \Delta \mathrm{N} 154$; and $(\mathrm{P}, \mathrm{D})$ added in $1 \mathrm{x} \Delta \mathrm{N} 169$.

Carboxy-terminal deletions of lexA-HAP2 were constructed in a similar manner by the addition of an $\mathrm{XbaI}$ 14-mer linker (NEB, 1062), which encodes a TAG nonsense codon in all three reading frames, at appropriate Klenow-blunted restriction sites of pSP64 : HAP2 as follows: PvuII (lx $\Delta \mathrm{C} 258)$, NheI $(\mathrm{lx} \Delta \mathrm{C} 230)$, DdeI (lx $\Delta \mathrm{C} 218), \quad M b o I I ~(l \mathrm{x} \Delta \mathrm{C} 208), \quad$ AluI (lx $\Delta \mathrm{C} 178), \quad$ ScaI

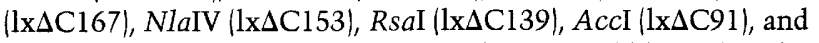
StyI ( $\mathrm{x} \Delta \mathrm{C} 45)$. These plasmids were also cut and blunted at the downstream NdeI site so that all HAP2-coding sequence downstream of the carboxy-terminal deletion endpoint was removed, except in the case of $\mathrm{lx} \Delta \mathrm{C} 197$, which was constructed by Klenow fill-in of a Hinfl site, as described above for $\Delta \mathrm{Cl} 197$. Reconstruction of these carboxy-terminal deletions as lexA fusions was described above except that a $B g l I I-E c o$ RI fragment encompassing the lexA-HAP $2 \Delta \mathrm{C}$ was used. Codons introduced at the carboxyl terminus of thee lexA-HAP $2 \Delta \mathrm{C}$ construct by the addition of the $X b a \mathrm{I}$ stop codon linker are as follows: $(\mathrm{A}, \mathrm{S}, \mathrm{L}, \mathrm{D})$ added in $\mathrm{lx} \Delta \mathrm{C} 258, \mathrm{~lx} \Delta \mathrm{C} 208$, and $\mathrm{lx} \Delta \mathrm{C} 178$; (S) added in $\mathrm{l} x \Delta \mathrm{C} 230$; (D) added in $\mathrm{lx} \Delta \mathrm{C} 218$; $(\mathrm{S}, \mathrm{S}, \mathrm{L}, \mathrm{D})$ added in $\mathrm{lx} \Delta \mathrm{C} 167$ and $\mathrm{lx} \Delta \mathrm{C} 139$; and $(\mathrm{L}, \mathrm{V})$ added in $\mathrm{lx} \Delta \mathrm{C} 153, \mathrm{~lx} \Delta \mathrm{C} 91$, and $1 \mathrm{x} \Delta \mathrm{C} 45$.

Codon insertion mutations of lexA-HAP2 were constructed from the appropriate codon insertion pSP64 : HAP2 derivatives described above. Double deletions (amino- and carboxy-terminal) of lexA-HAP2 were derivatives of $\Delta \mathrm{N} 141$ and $\Delta \mathrm{N} 154$ obtained by adding the $X b a \mathrm{I} 14$-mer stop codon linker at the same restriction sites as described for the corresponding single carboxy-terminal deletion constructs except in the case of $\mathrm{l} x \Delta \mathrm{N} 154 \Delta \mathrm{C} 197$, which was obtained from $\Delta \mathrm{N} 154$ by Klenow fill-in of a HinfI site and addition of the XbaI 14-mer linker. Codons introduced by added linker sequences at the lexA/ $\triangle$ HAP2 fusion and carboxyl terminus of double deletion constructs are as described above for the corresponding single deletion mutations except $\mathrm{lx} \Delta \mathrm{N} 154 \Delta \mathrm{C} 197$ in which the amino acid sequence $(S, S, L, D)$ is added at the carboxyl terminus.

\section{Construction of lexA-HAP4}

Fusion of lexA codons $1-87$ to HAP4 codons $10-554$ was 
achieved by ligating a BamHI 10-mer linker (NEB) to a StuI digest of pSLF406 (Forsburg and Guarente 1989a). Following digestion with SacI, blunting with Klenow, and restriction with BamHI, a 2-kb BamHI-SacI fragment carrying all but the first 9 codons of the HAP4 ORF was isolated and reconstructed into the BglII-EcoRI backbone of intermediate construct pSP64 : lexA-HAP2, as described above for the carboxy-terminal deletions of lexA-HAP2. The lexA-HAP4 fusion was released on a HindIII-HindIII fragment and reconstructed into yeast vector PAAH5 as described above for the lexA-HAP2 deletion constructs. The codon $(\mathrm{P})$ is added at the lexA-HAP4 fusion junction by linker sequence.

\section{Construction of HAP2-GAL4 acidic domain fusion}

Fusion of the carboxy-terminal 129 amino acids of GAL4HAP2 codon 258 was achieved as follows. A PvuII-PvuIl fragment, carrying GAL4 codons 753-881, was isolated from plasmid pRB1027 (Brent and Ptashne 1985). BamHI 12-mer linkers were ligated onto the ends and restricted with $\mathrm{BamHI}$. The resulting fragment was cloned into the $B a m H I$ site at the HAP2/lacZ fusion junction of pJP258 (Pinkham et al. 1987). Introduced linker sequence creates the codons $(A, R, S, A)$ between HAP2 amino acid 258 and GAL4 amino acid 753. Single inserts in each orientation were obtained, and these constructs were screened for complementation in hap2, hap 3 , and hap 4 null strains (described above). Only constructs in which the GAL4 acidic activation domain was fused to HAP2 resulted in a partial complementation in the hap4 null strain.

\section{Acknowledgments}

This work is supported by a National Institutes of Health (NIH) grant (GM30454) to L.G. and predoctoral training grants from NIH and Dupont to J.T.O.

The publication costs of this article were defrayed in part by payment of page charges. This article must therefore be hereby marked "advertisement" in accordance with 18 USC section 1734 solely to indicate this fact.

\section{References}

Ammerer, G. 1983. Expression of genes in yeast using the ADC1 promoter. Methods Enzymol. 101: 192-201.

Barany, F. 1985. Two-codon insertion mutagenesis of plasmid genes by using single-stranded hexametric oligonucleotides. Proc. Natl. Acad. Sci. 82: 4202-4206.

Berg, J.M. 1989. DNA binding specificity of steroid receptors. Cell 57: 1065-1068.

Brennan, R.G. and B.W. Matthews. 1989. The helix-turn-helix DNA binding motif. J. Biol. Chem. 264: 1903-1906.

Brent, R. and M. Ptashne. 1985. A eukaryotic transcriptional activator bearing the DNA specificity of a prokaryotic repressor. Cell 43: 729-736.

Chodosh, L., A. Baldwin, R. Carthew, and P. Sharp. 1988a. Human CCAAT-binding protein have heterologous subunits. Cell 53: 11-24.

Chodosh, L., J. Olesen, S. Hahn, A. Baldwin, L. Guarente, and P. Sharp. 1988b. A yeast and a human CCAAT-binding protein have heterologous subunits that are functionally interchangeable. Cell 53: 25-35.

Courey, A.J. and R. Tiian. 1988. Analysis of SPl in vivo reveals multiple transcriptional domains, including a novel glutamine rich activation motif. Cell 55: 887-898.
Curran, T. and B.R. Franza. 1988. Fos and jun: The AP-1 connection. Cell 55: 395-397.

Denis, C.L., J. Ferguson, and E.T. Young. 1983. mRNA levels for the fermentative alcohol dehyrogenase of Saccharomyces cerevisiae decrease upon growth on a nonfermentable carbon source. J. Biol. Chem. 258: 1165-1171.

Forsburg, S.L. and L. Guarente. 1988. Mutational analysis of upstream activation sequence 2 of the CYC1 gene of Saccha romyces cerevisiae: A HAP2-HAP3-responsive site. Mol. Cell Biol. 8: 647-654.

1989a. Identification and characterization of HAP4: A third component of the CCAAT-bound HAP2/HAP3 heteromer. Genes Dev. 3: 1166-1178.

$-1989 \mathrm{~b}$. Communication between mitochondria and the nucleus in regulation of cytochrome genes in the yeast Saccharomyces cerevisiae. Annu. Rev. Cell Biol. 5: 153-180.

Guarente, L. and T. Mason. 1983. Heme regulates transcription of the CYC1 gene of S. cerevisiae via an upstream activation site. Cell 32: 1279-1286.

Guarente, L., B. Lalonde, P. Gifford, and E. Alani. 1984. Distinctly regulated tandem upstream activation sites mediate catabolite repression of the CYCl gene of $S$. cerevisiae. Cell 36: $503-511$.

Hahn, S. and L. Guarente. 1988. Yeast HAP2 and HAP3: Transcriptional activators in a heteromeric complex. Science 240: $317-321$.

Hahn, S., J. Pinkham, R. Wei, R. Miller, and L. Guarente. 1988. The HAP3 regulatory locus of Saccharomyces cerevisiae encodes divergent overlapping transcripts. Mol. Cell Biol. 8: $655-663$.

Hai, T., F. Liu, W.J. Coukos, and M.R. Green. 1989. Transcription factor ATF cDNA clones: An extensive family of leucine zipper proteins able to selectively form DNA-binding heterodimers. Genes Dev. 3: 2083-2090.

Haldi, M. and L. Guarente. 1989. N-terminal deletions of a mitochondrial signal sequence in yeast. $\%$ Biol. Chem. 264: $17107-17112$.

Hope, I.A. and K. Struhl. 1986. Functional dissection of eukaryotic transcriptional activator protein, GCN4 of yeast. Cell 46: $885-894$.

Keng, T. and L. Guarente. 1987. Constitutive expression of the yeast $H E M 1$ gene is actually a composite of activation and repression. Proc. Natl. Acad. Sci. 84: 9113-9117.

Klug, A. and D. Rhodes. 1987. Zinc fingers: A novel protein motif for nucleic acid recognition. Trends Biochem. Sci. 12: $464-469$.

Kouzarides, T. and E. Ziff. 1989. Leucine zippers of fos, jun and GCN4 dictate dimerization specificity and thereby control DNA binding. Nature 340: 568-571.

Landschulz, W.H., P.F. Johnson, and S.L. McKnight. 1988. The leucine zipper: A hypothetical structure common to a new class of DNA binding proteins. Science 240: 1759-1764.

Lassar, A.B., J.N. Buskin, D. Lockshon, R.L. Davis, S. Apone, S.K. Hauschka, and H. Weintraub. 1989. MyoD is a sequence-specific DNA binding protein requiring a region of myc homology to bind to the muscle creatine kinase enhancer. Cell 58: 823-831.

Lech, K., K. Anderson, and R. Brent. 1988. DNA-bound Fos proteins activate transcription in yeast. Cell 52: 179-184.

Leszczynski, J.F. and G.D. Rose. 1986. Loops in globular proteins: A novel class of protein secondary structure. Science 234: 849-855.

Ma, J. and M. Ptashne. 1987a. Deletion analysis of GAL4 defines two transcriptional activating sequences. Cell 48: 847-853.

Mermod, N., E.A. O'Neill, T.J. Kelly, and R. Tjian. 1989. The 
proline-rich transcriptional activator of CTF/NF-1 is distinct from the replication and DNA binding domain. Cell 58: $741-753$.

Murre, C., P.S. McCaw, and D. Baltimore. 1989a. A new DNA binding and dimerization motif in immunoglobulin enhancer binding, daughterless, MyoD, and myc proteins. Cell 56: 777-783.

Murre, C., P.S. McCaw, H. Vaessin, M. Caudy, L.Y. Jan, Y.N. Jan, C.V. Cabrera, J.N. Buskin, S.E. Hauschka, A.B. Lassar, H. Weintraub, and D. Baltimore. 1989b. Interactions between heterologous helix-loop-helix proteins generate complexes that bind specifically to a common DNA sequence. Cell 58: 537-544.

Neuberg, M., M. Schuermann, J.B. Hunter, and R. Muller. 1989. Two functionally different regions in Fos are required for the sequence-specific DNA interaction of the Fos/Jun protein complex. Nature 338: 589-590.

Olesen, J.T., S. Hahn, and L. Guarente. 1987. Yeast HAP2 and HAP3 activators both bind to the CYC1 upstream activation site UAS2 in an interdependent manner. Cell 51: 953-961.

Pabo, C.O. and R.T. Sauer. 1984. Protein DNA recognition. Annu. Rev. Biochem. 53: 293-321.

Pfeifer, K., K.S. Kim, S. Kogan, and L. Guarente. 1989. Functional dissection and sequence of yeast transcriptional activator HAPl. Cell 56: 291-301.

Pinkham, I.L. and L. Guarente. 1985. Cloning and molecular analysis of the HAP2 locus: A global regulator of respiratory genes in Saccharomyces cerevisiae. Mol. Cell Biol. 5: 34103416.

Pinkham, J.L., J.T. Olesen, and L. Guarente. 1987. Sequence and nuclear localization of the Saccharomyces cerevisiae HAP2 protein, a transcriptional activator. Mol. Cell Biol. 7: 578585.

Ptashne, M. 1988. How eukaryotic transcriptional activators work. Nature 335: 683-689.

Rothstein, R.J. 1983. One-step gene disruption in yeast. Methods Enzymol. 101: 202-245.

Sambrook, I., E.F. Fritsch, and T. Maniatis. 1989. Molecular cloning: A laboratory manual, 2nd ed. Cold Spring Harbor Laboratory Press, Cold Spring Harbor, New York.

Schneider, J.C. 1989. "Coordinate control of cytochrome genes." Ph.D. thesis. M.I.T., Cambridge, MA.

Scott, M.P., J.W. Tamkun, and G.W. Hartzell III. 1989. The structure and function of the homeodomain. Biochim. Biophys. Acta 989: 25-48.

Sherman, R., G.R. Fink, and J.B. Hicks. 1986. In Methods in yeast genetics. Cold Spring Harbor Laboratory, Cold Spring Harbor, New York.

Wharton, K.A., B. Yedvobnick, V.G. Finnerty, and S. AvtavanisTsakonas. 1985. opa: A novel family of transcribed repeats shared by the notch locus and other developmentally regulated loci in D. melanogaster. Cell 40: 55-62.

Wiederrecht, G., D. Seto, and C.S. Parker. 1988. Isolation of the gene encoding the $S$. cerevisiae heat shock transcription factor. Cell 54: $841-853$. 


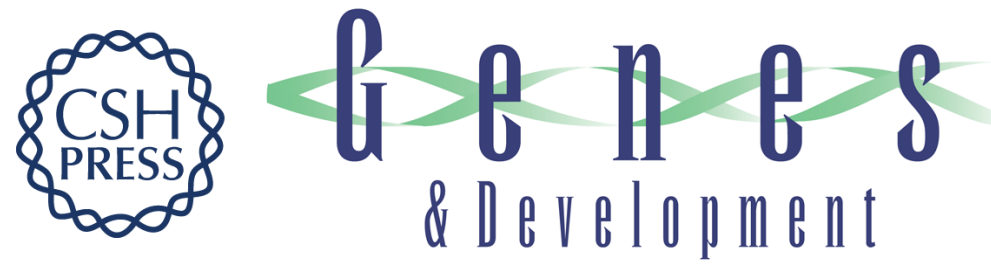

\section{The HAP2 subunit of yeast CCAAT transcriptional activator contains adjacent domains for subunit association and DNA recognition: model for the HAP2/3/4 complex.}

\section{J T Olesen and L Guarente}

Genes Dev. 1990, 4:

Access the most recent version at doi:10.1101/gad.4.10.1714

References This article cites 42 articles, 14 of which can be accessed free at:

http://genesdev.cshlp.org/content/4/10/1714.full.html\#ref-list-1

License

Email Alerting Service

Receive free email alerts when new articles cite this article - sign up in the box at the top right corner of the article or click here.

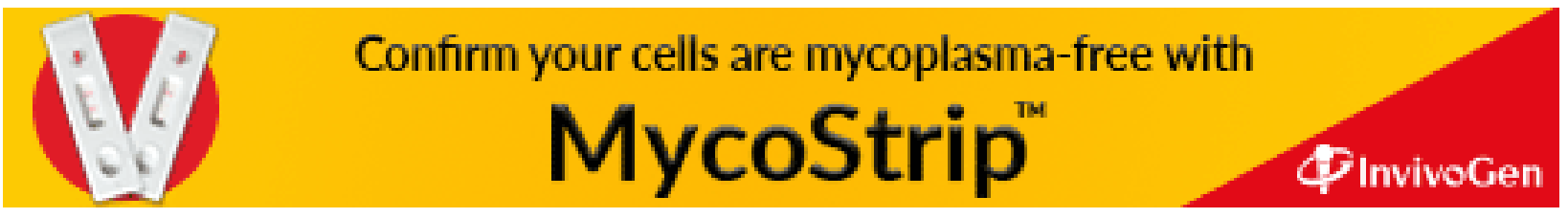

